\title{
An Effective Data Access Method for Public Cloud Data Storage
}

\author{
M.Laxmaiah, Teja, M.Narendra
}

\begin{abstract}
These days attribute-based encryption has been gotten as a promising strategy to give versatile and secure data to oversee the conveyed storage in the cloud registering environment. In the attribute-based encryption plan, the single property pro should execute the customer legitimacy check and mystery key flow, and thusly, its outcomes. Customers may be stuck in the believing that at a stretch get their mystery keys and choose to get the required data from Cloud. So as to maintain a strategic distance from the single-point execution bottleneck, we are proposing multi-master access control plan which will assess the issue such way that it gives increasingly successful access control on cloud data to the clients.
\end{abstract}

Keywords: Cloud Storage, Central Authority, Attribute Authority, Data Security

\section{INTRODUCTION}

In late time, cloud processing has picked up a great deal of prominence in the figuring worldview. The most significant thing in cloud figuring is the appropriated storage organization [13][14].The use of dis-tributed storage system demonstrating clients more transparency, higher quality and more ensured confirmation for client data and its security. The fundamental issue in the open cloud is giving data by cloud-based associations, who are for uncovering their data in space of data owners. The ordinary access control procedures in the customer/server architecture are not appropriate for dispersed storage in a cloud figuring environment. The data gets the chance to control in conveyed storage has transformed into a significant testing issue. To address this, there have been numerous plans proposed, among them the figure content policy attributebased encryption is seen as a most promising strategy. An amazing segment of figure content policy based encryption is that it blessings data owners arrange control subject to get to strategies which give progressively versatile and secure access control for distributed storage structure.

In cryptography, the client passage control is practiced by utilizing attribute-based encryption methods, where an owner's in-development is disordered with some structure over characteristics and a customer's lord key is set apart with its own qualities. Taking the properties of the customer's mystery key enables him to go into the framework and can the customer disentangle the contrasting

Revised Manuscript Received on September 10, 2019.

M.Laxmaiah, Department of Computer Science and Engineering, CMR Engineering College, Medchal (D), Hyderabad, Telangana, India (E-mail: laxmanmettu.cse@gmail.com)

Teja, Department of Computer Science and Engineering, CMR Engineering College, Medchal (D), Hyderabad, Telangana, India

(E-mail: tejamrecw1212@gmail.com)

M.Narendra, Department of Computer Science and Engineering, CMR Engineering College, Medchal (D), Hyderabad, Telangana, India

(E-mail: narendramupparaju06@gmail.com) security to the data. Since the circulated storage is overseen

ciphertext with get the plaintext. Thus now, the ciphertext based access control systems for the dispersed storage in the cloud have been made for two significant arrangements, for example, single master access control [5][9] and multimaster access control [10][12]. Despite the fact that there many figure content based access control plans with a great deal of appealing highlights however none of them are neither effective nor hearty in the age of the key. In this manner, in transgression authority plans, just a single authority is accessible as an in control to make all emit key solicitations inaccessible during the data get to period and in the multi-authority plot additionally exist the issue, since every one of numerous authority needs to deal with the distinctive attribute set of the clients. In single-authority verification plots, the just a single authority must check the legitimacy of clients' attributes before creating mystery keys. As the entrance control framework is related with data security. The legitimate client who have his mystery key related with his own highlights with age, date of birth. The key issuing is the constant procedure for fulfill ing these necessities.

Be that as it may, in reality, the attributes of every client is contrast ent. To manage the check of different highlights, the client might be required to be available confirmation. Furthermore, the impact of the transgression gle-point bottleneck can be consolidated to a specific sum. However, this arrangement will bring out dangers to security issues. Along these lines, there are various practically undistinguishable specialists playing out a similar technique, it is elusive the dependable authority if missteps have been made during the time spent mystery key age and dispersion.

In this paper, we separate the procedure of customer authenticity con-firmation from his mystery key, individual attributes, for example, age, sexual orientation, and job. From these two systems, numerous specialists are advanced. Here, we characterized various masters, explicitly property specialists as Attribute Authority (AA), all of them is in charge of the en-tire list of capabilities and can deal with the customer realness check auto-nomously. Similarly, there is an authority in particular, Central Au-thority (CA) in charge of the mystery key age and its circula-tion. Before playing out a mystery key age and course process, one of the AAs is picked to affirm the legitimacy of the customer's characteristics and it makes a transitional emit key and sends it to $\mathrm{CA}$. He delivers the mystery key for the customer dependent on the procured transitional key, with no need of any more confirmation further. By these lines, a few AAs can work in parallel to share the store of the dull procedure 
of realness confirmation and save for each other so as to oust the single-point bottleneck on execution. Henceforth it ace vides greater security for the data access from the cloud.

In this paper, subsection 2 depicts the related research work conveyed around there and secure validation framework in circulated cloud processing is portrayed in subsection 3 . The subsections 4 and 5 briefs up about the validation procedure and results from examination respectively. Subsection 6 finishes up the paper.

\section{RELATED WORK}

In cloud processing, accessible encryption conspire over redistributed data is a hot research theme for fascinating analysts. Nonetheless, a large portion of the current plans on scrambled pursuit over redistributed cloud data pursues the model for all sizes of data and disregard customized search data of the clients. Be that as it may, the greater part of them bolster just careful watchword search, which extraordinarily influences data ease of use and client expe-rience. So it is an incredible moving errand to us, how to plan an accessible encryption plot that supports customized search and improves the client search involvement. In this work, they tackled the issue of customized multi-catchphrase positioned search over scrambled data while safeguarding security in cloud figuring [1]. With the assistance of semantic philosophy wordnet, they manufactured a client intrigue model for an individual client by breaking down the client's hunt history, and embrace a scoring system to express client intrigue shrewdly. To address the constraints of the model and catchphrase accurate hunt, they are prosed plans for various inquiry aims. Broad tests on genuine world dataset approved and broke down.

The expanding appropriation of cloud registering has developed as various individuals redistribute their datasets into a cloud. The datasets typically are scrambled before redistributing to save the security [2]. In any case, the normal practice is to make the data encoded and after that search the datasets with given catchphrases. Numerous plans are proposed to make encoded data accessible dependent on watchwords. Be that as it may, watchword based hunt plans overlook the semantic portrayal infor-mation of client's and can't meet totally clients search aim. In this way, how to structure a substance based hunt plan and make se-mantic inquiry increasingly successful and setting mindful is a troublesome test. In their work, have proposed a creative semantic pursuit plan dependent on the idea chain of importance and the semantic connection between ideas in the scrambled datasets. All the more explicitly, this plan initially records the archives and fabricates trapdoor dependent on the idea hie-rarchy. To further improve the pursuit productivity, they use a tree-based file structure to arrange all the report list vectors. In test results dependent on this present reality, datasets demonstrate the plan is more proficient than past plans.

With the prevalence of gathering data partaking in open cloud processing, the protection and security of gathering sharing data have turned into a noteworthy issue [6]. The cloud specialist co-op may not be treated as a confided in outsider in view of its semi-trust nature, and in this manner the conventional se-curity models can't be direct summed up into cloud-based gathering sharing structures. P.Hang, etl, proposed a novel secure gathering sharing structure for open cloud [3], which can adequately take advertisement vantage of the cloud servers' assistance however have no delicate data being ex-presented to assailants and the cloud specialist co-op. The system com-bines intermediary mark and intermediary re-encryption together into a convention. By applying the intermediary signature method, the gathering head can effec-tively award the benefit of gathering the board to at least one picked bunch individuals. The upgraded Tree-Based Group Diffie-Hellman plan empowers the gathering to arrange and refresh the gathering key sets with the assistance of cloud servers, which does not require the majority of the gathering individuals been online constantly. By embracing intermediary re-encryption, most computationally escalated tasks can be designated to cloud servers without revealing any private data. Broad security and execution examination demonstrate that their proposed plan is more ef-ficient and fulfills the security necessities for open cloud-based secure gathering sharing.

The scientist, Y. Wu .etl,exhibits a novel multi-message ci-phertext policy attribute-based encryption framework[4] and use plan of a passage control plot for sharing valuable media in light of infor-mation single-point execution bottleneck purchasers' characteristics[7][8], for example, age, nationality, or sex rather than a reasonable check of the purchase ers' names. The arrangement is adaptable in light of the way that multi-message ciphertext policy attributebased encryption draws in a substance sup-plier to display away system and scramble various messages inside one ciphertext with the certified objective that simply the customers whose qualities satisfy the manner in which approach can disentangle the Ciphertext.

With the ongoing appropriation and conveyance of the data sharing worldview in dispersed systems[15], for example, online informal communities or cloud registering, there have been expanding requests and worries for circulated data security. One of the most testing issues in data sharing frameworks is the authorization of access arrangements and the help of strategies refreshes. Ciphertext-policy attribute-based encryption [9] is turning into a promising cryptographic answer for this issue. This empowers data proprietors to characterize their own entrance strategies over client attributes and authorize the arrangements on the data to be dispersed. In any case, the advan-tage accompanies a noteworthy disadvantage which is known as a key bond prob-lem. The key age focus could decode any messages routed to explicit clients by producing their private keys.

\section{OUR PROPOSED APPROACH}

In this segment, we proposed a safe validation model for the bar lic cloud with various parts, for example, a central authority, different attribute specialists (AA), data proprietors (DOs), data clients and cloud specialist co-ops (to be specific Amazon, Google, Microsoft, Salesforce, and so on.) with numerous cloud servers. In our proposed plan, we have given five unique assignments and every single one 
of them can play out an alternate undertaking. The jobs and duties of these specialists are clarified in the accompanying lines.

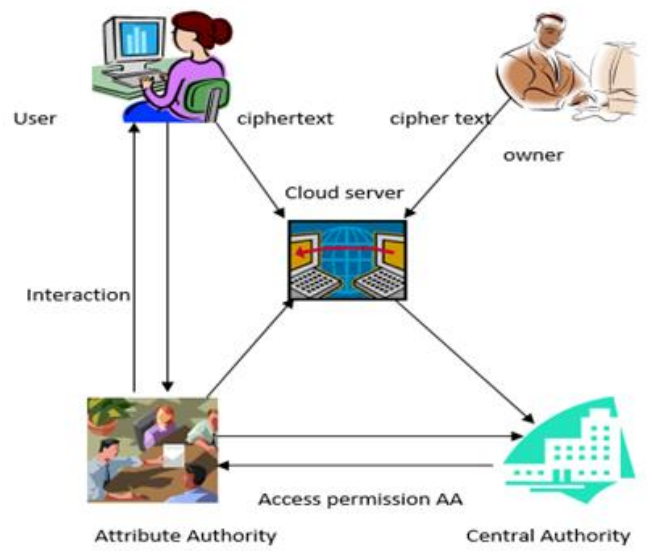

\section{Fig. 3.1.The architecture of distributed Cloud Computing}

Cloud Servers are kept up by the cloud specialist organization through on the web. The cloud servers are in charge of executing the right now allocated assignments for benefits. The server gathers all the transferred records and the mystery data of the data proprietors. These servers give the data storage and data conveyance dependent on the client's solicitation.

Central Authority is the manager of the whole framework and is for the framework development by setting up the framework parameters. He likewise in charge of creating an open key for every client dependent on his attributes. The CA is completely controlling authority which will be on the web and accepted as a completely confided in part. It won't enable any elements to procure its data without checking its login certifications. CA is in charge of social event the clients emit data based on AA input. CA has the authority to survey which AA has given illicit real data about the clients.

The attribute authorities are in charge of performing client authenticity check and creating transitional keys for authenticity confirmed clients. Dissimilar to a large portion of the current multi-authority plans where every AA deals with a disjoint attribute set individually, our proposed plan includes different specialists to share the duty of client authenticity confirmation and every AA can play out this procedure for any client freely. At the point when an AA is chosen, it will check the clients' genuine attributes by difficult work or verification conventions, and create a transitional key related with the attributes that it has authenticity confirmed. The middle key is another idea to help CA to produce keys.

The data owner characterizes the entrance policy about who can gain admittance to each record transferred in the cloud, and encodes the document under the characterized policy. By utilizing symmetric encryption calculation every proprietor encodes the data and the proprietor casings get to policy dependent on attribute set and scrambles the symmetric key under the policy for open keys acquired from CA. From that point onward, the proprietor sends the entire encoded data and the en-crypted symmetric key (ciphertext to the cloud server) to be put away in the cloud.

The data user is doled out a worldwide client character by CA. The client has a lot of attributes and is furnished with a mystery key related with his attribute set. The client can openly get any intrigued scrambled data from the cloud server. Nonetheless, the client can unscramble the scrambled data if and just if his/her attribute set fulfills the entrance policy implanted in the encoded data.

\section{THE PROCESS OF DATA ACCESS AUTHENTICATION IN PUBLIC CLOUD}

The Data proprietor and Data client can sign in utilizing their particular creden-tials through a cloud. When the data client qualifications are confirmed, the client can get to the data from the cloud. AA can distinguish all infor-mation by the clients which are accommodated cloud and ready to create transitional keys for authenticity checked clients. The $\mathrm{CA}$ is completely control-ling authority which will be on the wand producing an open key for every client dependent on their attributes.

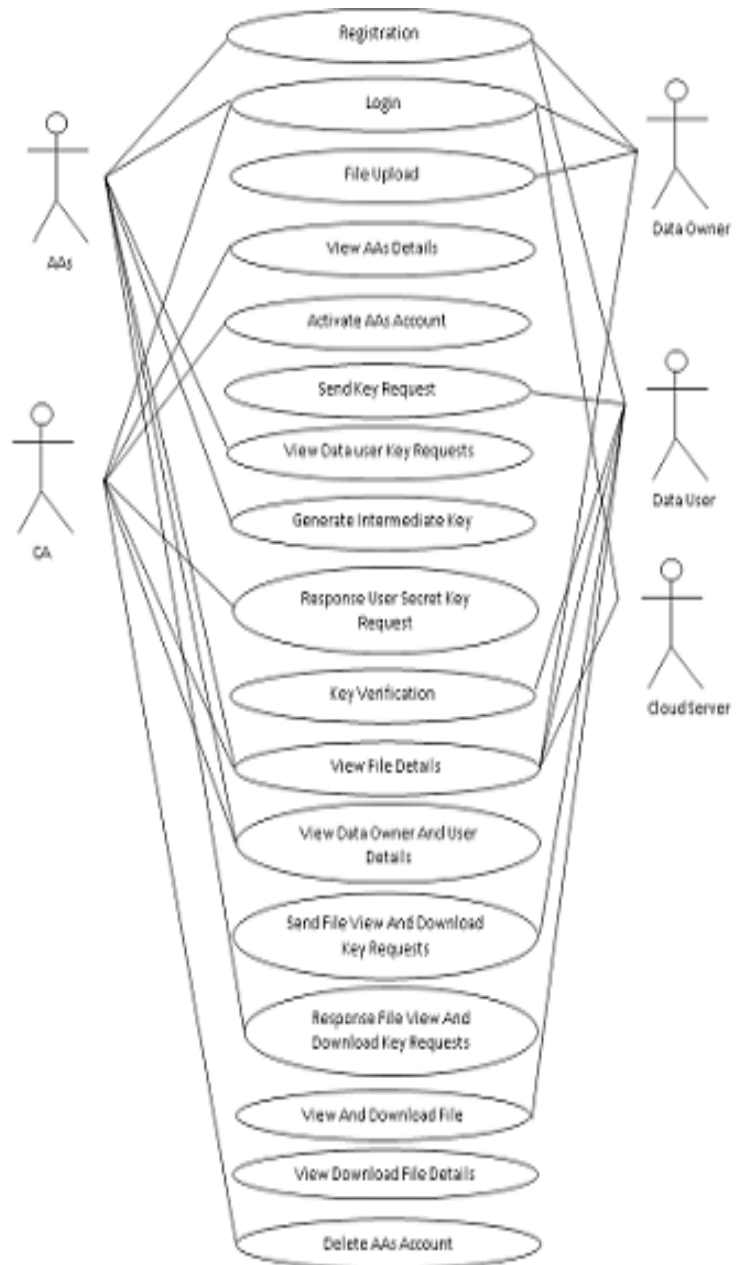

Fig.4.1. Cloud Data Access Authentication 


\section{RESULTS}

In this segment, hypothetical and experimental investigation is clarified. The chart is drawn between the normal holding up times versus the landing rate. Entry time signifies the pace of a solicitation of the client. Various attribute specialists indicate the quantity of clients hanging tight in the line for the reaction from the proprietor. From figure 6.1 , we can see that the normal holding up time increments quickly with the expansion of landing rate when the entry rates are low. Be that as it may, later the normal holding up time will wind up consistent in light of the fact that recently landing clients will be dismissed by the framework because of the breaking point length of holding up line. In spite of the fact that utilizing all the more working AAs brings bigger arrangement cost, by joining the

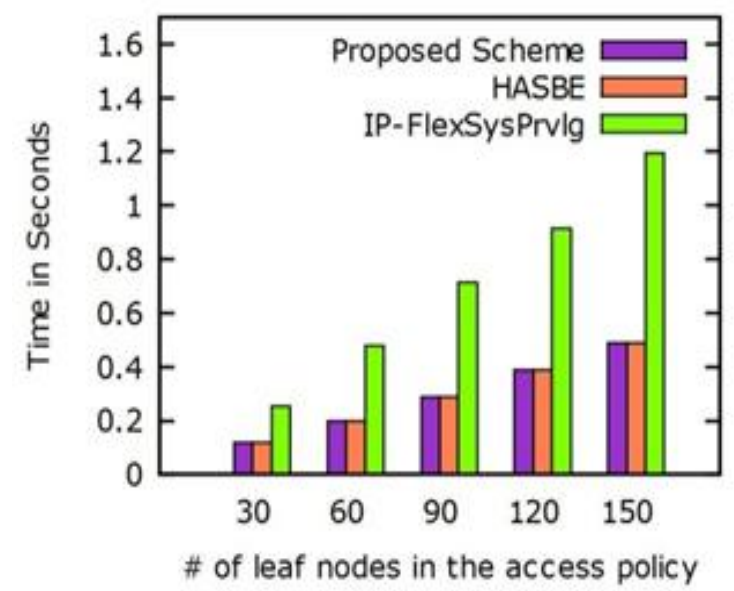

Fig .6.1. The time required for Encryption

disappointment rate and the normal holding up time, we can guarantee that the confi-guration of various AAs can furnish mystery key age administration with high caliber just as minimal effort

Encryption: In the proposed technique, records are scrambled dependent on the entrance policy. Limit esteems are accessible at non-leaf hubs of access policy and attributes are speaking to the leaf hubs. Time is taken for encoding a record as indicated by the entrance policy and various leaf hubs in the entrance tree. Fig 6.1 delineates the time required for encryp-tion by shifting the quantity of leaf hubs of access policy attributes.

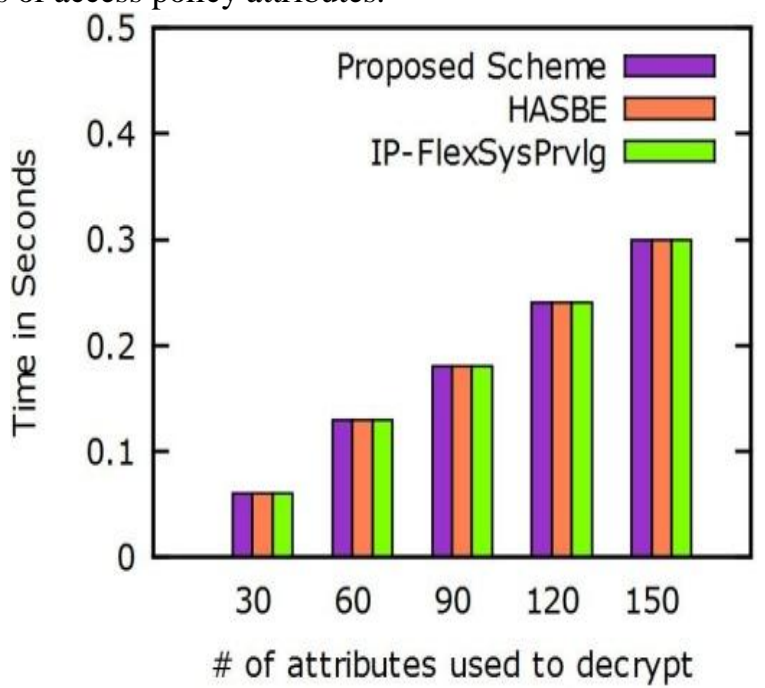

Fig. 6.2. The time required in decryption
Decryption: In the above figure 6.2, indicated time required for unscrambling can be de-termined by dependent on the quantity of leaf hubs of access policy. The time required to decode the record by fluctuating the leaf hubs given as 30,60, 90, 120, and 150. The Time required for decoding a record for various plans continues as before. The Time required for encryption or decoding of a record develops directly with the expansion in various leaf hubs of access tree. We saw that the trial results confirm our hypothetical investigation.

\section{CONCLUSIONS}

In this paper, we broke down the attribute-based encryption system to wipe out the single-point execution bottleneck of the current plans. We directed point by point security and execution examination to confirm that our plan is secure and productive. We attempt to re-duce the repetition of the conveyed pieces more than a few servers on the cloud. Along these lines we have accomplished an improved data storage. We likewise gave simple relocation of data between various cloud specialist co-ops by composing fitting API for interoperability. To overhaul the framework for item and square storage which supports interoperability of data. As cloud processing and IoT innovations are adding to the exponential development of enormous data in future correspondence as an administration may likewise bolster item and square storage for fulfillment reason.

\section{REFERENCES}

1. 1.Z. Fu, K. Ren, J. Shu, X. Sun, and F. Huang, "Empowering customized look over Outsourced information with productivity change," IEEE Transactions on Parallel\& Distributed Systems, vol.127, no.9, pp.2546-2559, (2016).

2. Z. Fu, X. Sun, S. Ji, and G. Xie, "Towards productive substance mindful inquiry over scrambled outsourced information in the cloud," in Proceedings of 2016 IEEE Conference on Computer Communications (INFOCOM 2016). IEEE, pp. 1-9, (2016).

3. 3.K. Xue and P. Hong, "A dynamic secure gathering sharing system out in the open distributed computing," IEEE Transactions on Cloud Computing, vol. 2, no. 4 pp. 459-470,( 2014).

4. $4 \mathrm{Y}$. Wu, Z. Wei, and H. Deng, "Ascribe based access to adaptable media in cloud-helped content sharing", IEEE Transactions on Multimedia, vol. 15, no. 4, pp. 778- 788, (2013).

5. 5.J. Hur, "Enhancing security and proficiency in attribute-based information sharing," IEEE Transactions on Knowledge and Data Engineering, vol. 25, no. 10, pp. 2271-2282, (2013).

6. 6 J. Hur and D. K.Noh, "Quality-based access control with proficient repudiation in information outsourcing frameworks," IEEE Transactions on Parallel and Distributed Systems, vol. 22, no. 7, pp. 1214- 1221, (2011).

7. 7 J.Hong, K.Xue, W.Li, andY.Xue, "TAFC: Time and characteristic variables consolidated access control on time touchy information in broad daylight cloud," in Proceedings of 2015IEEE Global Communications 
Conference (GLOBECOM2015). IEEE, pp. 1-6, (2015)

8. 8 Y. Xue, J. Hong, W. Li, K. Xue, and P. Hong, "LABAC: An area mindful property-based access control conspire for distributed storage," in Proceedings of 2016 IEEE Global Communications Conference (GLOBECOM 2016). IEEE, pp. 1-6, (2016).

9. 9 A. Lewko and B. Waters, "Decentralizing property based encryption", in Advances in CryptologyEUROCRYPT2011. Springer, pp. 568-588, (2011).

10. $10 \mathrm{~K}$. Yang, X. Jia, K. Ren, and B. Zhang, "DACMACS: Effective information get to control for multiexpert distributed storage frameworks," in Proceedings of 2013 IEEE Conference on Computer Communications (INFOCOM 2013). IEEE, pp. 2895- 2903, (2013)

11. 11 J. Chen and H. Mama, "Effective decentralized attribute based get to control for distributed storage with client denial," in Proceedings of 2014 IEEE International Conference on Communication (ICC2014), pp. 37823787.

12. 12 M. Pursue and S. S. Chow, "Enhancing protection and security in multi-expert trait based encryption," in Proceedings of the sixteenth ACM meeting on Computer and Communications Security (CCS 2009). ACM, pp. 121-130,(2009).

13. 13 M.Lippert, E.G. Karatsiolis, A. Wiesmaier, and J. A. Buchmann, "Registry based enrollment in broad daylight key foundations." in Proceedings of the Fourth International Workshop for Applied PKI(IWAP2005), pp.17-32,(2005)

14. 14 W. Li, K.Xue, Y.Xue, and J. Hong, "TMACS: A vigorous and evident edge multi-expert access control framework in broad daylight distributed storage," IEEE Transactions on Parallel and Distributed Systems, vol. 27, no. 5, pp. 1484-1496, 2016.

15. Kaiping Xue, Jianan Hong, Yingjie Xue, David S.L. Wei, Nenghai Yu, Peilin Hong, "CABE: A New Comparable Attribute-Based Encryption Construction with 0Encoding and 1-Encoding", Computers IEEE Transactions on, vol. 66, no. 9, pp. 1491-1503, 2017. 